\title{
Civil engineering structure daily monitored through IR Thermography and environmental measurement.
}

\author{
by A. Criniere ${ }^{1, \star}$, J. Dumoulin ${ }^{1,4}$, L. Perez ${ }^{2}$ and F. Bourquin ${ }^{3}$
}

1 LUNAM Université, IFSTTAR, COSYS/SII, F-44340, Bouguenais, France, antoine.criniere@ifsttar.fr

2 LUNAM Université, LTN, UMR-CNRS 6607, rue Christian Pauc, 44306 Nantes, France, laetitia.perez@univ-nantes.fr

${ }^{3}$ Université Paris-Est, IFSTTAR, Cosys, F-77447, Marne-la-Vallée, France, frederic.bourquin@ifsttar.fr

${ }^{4}$ I4S team, INRIA Rennes, Campus de Beaulieu, 35042 Rennes cedex, France, iean.dumoulin@ifsttar.fr

\begin{abstract}
An integrated infrared measurement system synchronized with weather parameter measurements developed to make long term thermal monitoring of structural elements over a year is presented. The software and hardware architecture has been designed with the aim to be fully autonomous. Twin reinforced concrete beams, a damage one and a healthy one, are surveyed with this system. This study presents results obtained during the first six month of monitoring. Results obtained are detailed and discussed. A comparison with thermal measurements carried out inside the healthy beam is also proposed and discussed by comparison with external measurements.
\end{abstract}

\section{Introduction}

With the constant increase of the road traffic coupled with the aging of transport infrastructure, studying and developing robust system which allows to monitor and assess those structures is of growing interest. Among the techniques used [1], thermal monitoring with infrared thermography appears to be a good compromise between a nonintrusive method and possible added value after post-processing of acquired data. Through the past decade studies have shown the ability to monitor concrete and asphalt structure by active IR thermography [2] [3]. On site measurement using passive thermography have also been studied, by applying qualitative methods [4] and quantitative one [5]. These methods have been used to perform punctual control of various duration (few hours to few days).

Within the European project ISTIMES, whose main objective was to evaluate the potentialities offered by the integration of different electromagnetic techniques able to perform non-invasive diagnostics for the surveillance and monitoring of transport infrastructures [6], an information system, has been designed to perform very long term thermal monitoring of structures exposed to environmental condition, as the solar heat flux, natural convection or seasonal perturbation. This system called "IrLaW" is based on a multi sensing approach. It connects and synchronise information acquired by a weather station, a GPS and an infrared camera. It is fully integrated in a TCP/IP network [7]. In a previous study the system was used to assess a bridge deck without traffic disruption and shown the feasibility of the control procedure to detect and characterize the inner structure of the deck [8]. In the continuity of this former study, two scale 1 concrete beams have been submitted to environmental perturbation for over three years now. One of these beams has been damaged by high energetic mechanical impacts [9] and the other one, considered as healthy, was internally instrumented during its building.

Integrated in the Sensorbox hardware architecture by mid-2013, the "IrLaW" system has been set up to monitor those concrete beams over a year. Connected to weather station VAISALA WXT 520 and a FLIR SC 325 IR camera, the software and hardware architecture has been upgraded with the aim to be fully autonomous. The data are saved periodically and the system has its own power strategy including a failover power supply. At the healthy beam level, instrumentation provides information about the internal temperature field which can be used to check the measurement system and enhance a thermal model design.

This study presents results obtained after several months of measurements. The experimental procedure and the system architecture are also described. Infrared, weather and inside beam thermocouples measurements are presented, analysed and discussed.

\section{Measurement system overview}

The IrLaW (Infrared Through Lan and Wifi) software was initially designed for non-destructive testing by active infrared thermography using uncooled IRFPA camera in controlled environments (e.g. laboratory ones). From the ISTIMES [6] project IrLaW has been upgraded to propose a turnkey installation and new functionalities able to perform long term multi sensing data acquisition (see. figure 1) in outdoor conditions with OGC Standards compliance. This improvement is so called "SensorBox". It provides to the user, meteorological data synchronized with infrared monitoring and timing with GPS synchronisation. Data can be qualitative (Raw Data) but also quantitative (Using radiometric heat balance corrections on-line or off-line) thanks to the possibility to develop embedded physical model using a model compiler [7]. Raw data pass through a circular buffer in which the system acquires the raw data in accordance to a scenario which can be specialized for each application. All the data acquired are stored into a standard data structure. 


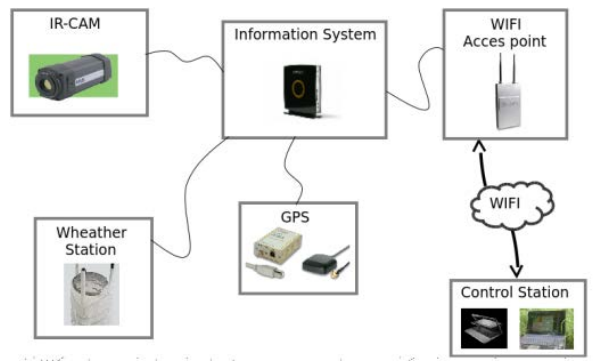

Fig. 1 IrLaW software architecture overview

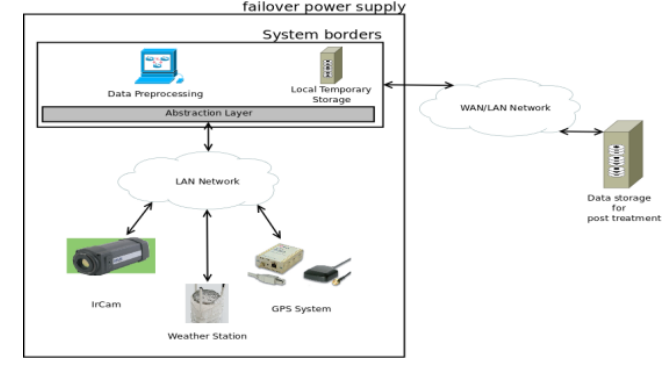

Fig. 2 SensorBox adapted architecture

The measurement system can be remotely control via WiFi connection which can be useful for maintenance or control operations on real site. A former study shows the capacity of the system to provide information over days [8] on a bridge opened to traffic.

In the present study the SensorBox has been improved and adapted (see figure 2) in order to reach full autonomy in term of power supply, very long term measurement capability (at least 1 year), and automated data base feeding. The power autonomy of the whole system is performed by a $12 \mathrm{~V}$ external battery of $102 \mathrm{Ah}$, which is enough to insure the system continuity for at least few days. The failover power supply system is also designed as a sensor, each hour the system check the battery level, and below a fixed threshold the SensorBox is safely turned off to avoid corrupted data.

The system controls a FLIR SC325 uncooled microbolometric IR camera with a 320X240 Focal Plane Array detector in band III, a weather station VAISALA WXT520 and a GPS UBLOX. The IR camera native frame rate is tuned to $25 \mathrm{~Hz}$. The acquisition scenario averages IR images with a sample rate of $0.1 \mathrm{~Hz}$ (including weather and GPS information) and saves them each hour into the system local hard drive. Each morning a batch on the data storage server retrieves data from the previous day and then flushes the internal system memory. As all the measured parameters are saved, this procedure avoids to overload the network and data loss in case of sudden stop of the system. For instance one full month of data acquisition represents an amount of about 40 Giga bytes saved in the server.

In the next section experimental benchmark and system implementation is presented and developed.

\section{Reinforced concrete beams and outdoor experiments set-up}

The SensorBox system presented below is used to monitor structural elements of scale 1 . These elements consist in two reinforced concrete beams ( $16 \mathrm{~m}$ long $\times 1 \mathrm{~m}$ height $\times 0.5 \mathrm{~m}$ width and 21 tons weight). One of these two twin beams has been instrumented with 48 thermocouples and 10 displacement gauges (see figure 3 ).
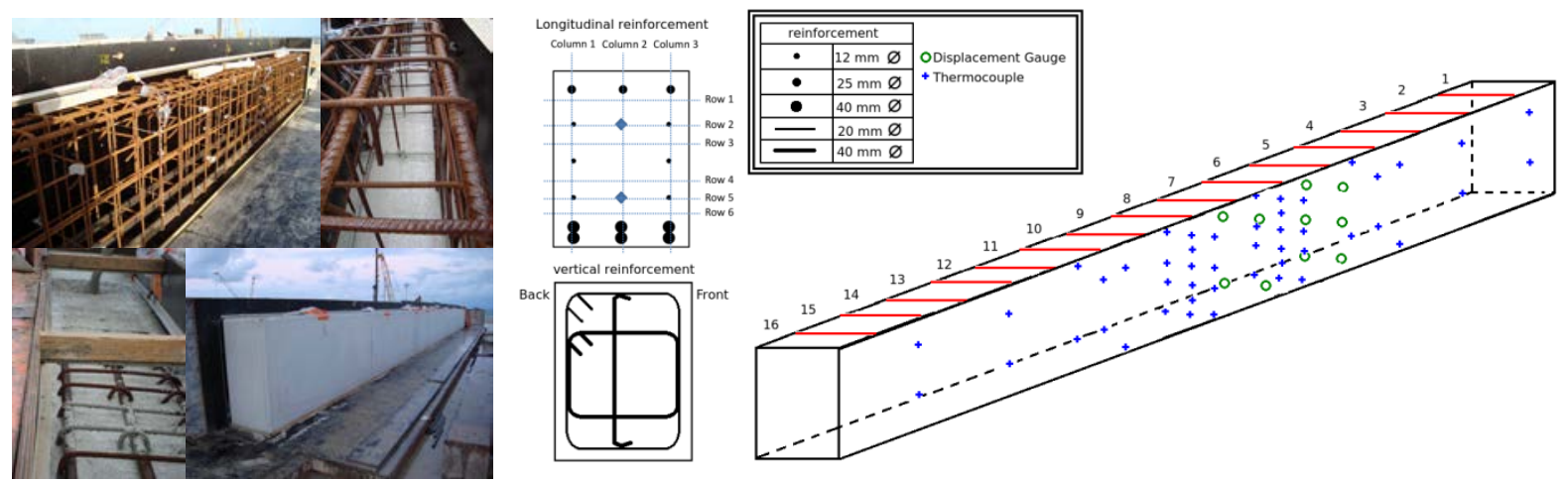

Fig. 3 Views of the instrumented beam during building (left) - Schemes of the instrumentation distribution (right)

The 48 thermocouples have been placed in order to allow 3D thermal field reconstruction in a further step of this study by using the approach developed in [10]. Each sensor is identified (tagged) by its row, column and section number.

Its twin sister has been damaged by several mechanical impact of high energy at the IFSTTAR falling rocks test station located in the French Alpes (see figure 4). To damage this beam, a 2.5 tons steel ball has been dropped on the concrete beam from various heights. A total of four mechanical impacts have been carried out on the upper face of the beam, three from a height of one meter and one from five meters [9]. The figure 4 right present the damage generated on the top of the beam at the end of the experiment. 

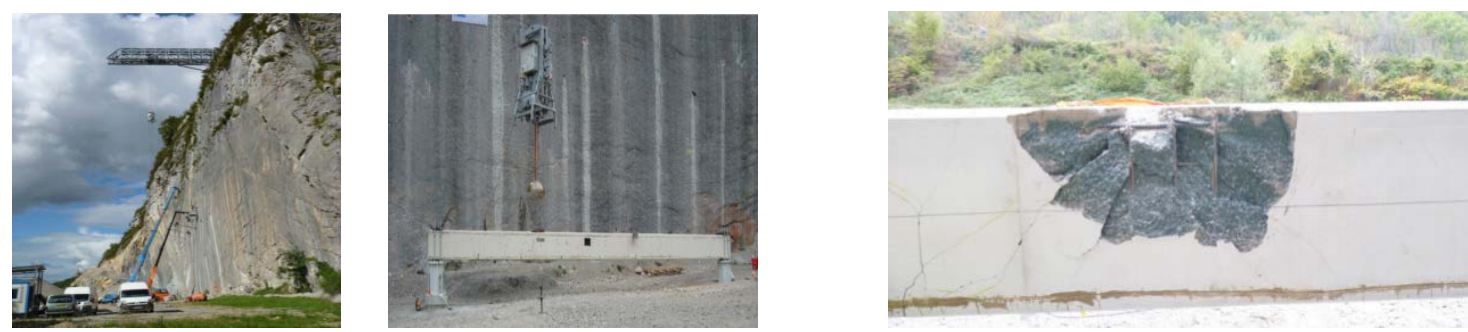

Fig. 4 IFSTTAR falling block test station (left), load of $2.5 \mathrm{~T}$ used (center), impact view (right)

The impacted area presents, in the rear face of the beam, a leaving of materials that allow to see the rebars. Analysis of the rebar positioning after each impact was presented in [9]. After this mechanical experiment, this damage beam was moved back from French Alpes to Nantes and placed behind and parallel to the longitudinal axis of its instrumented twin. The long term monitoring system was then set-up at this IFSTTAR large scale testing facilities in Nantes (see figure 5).
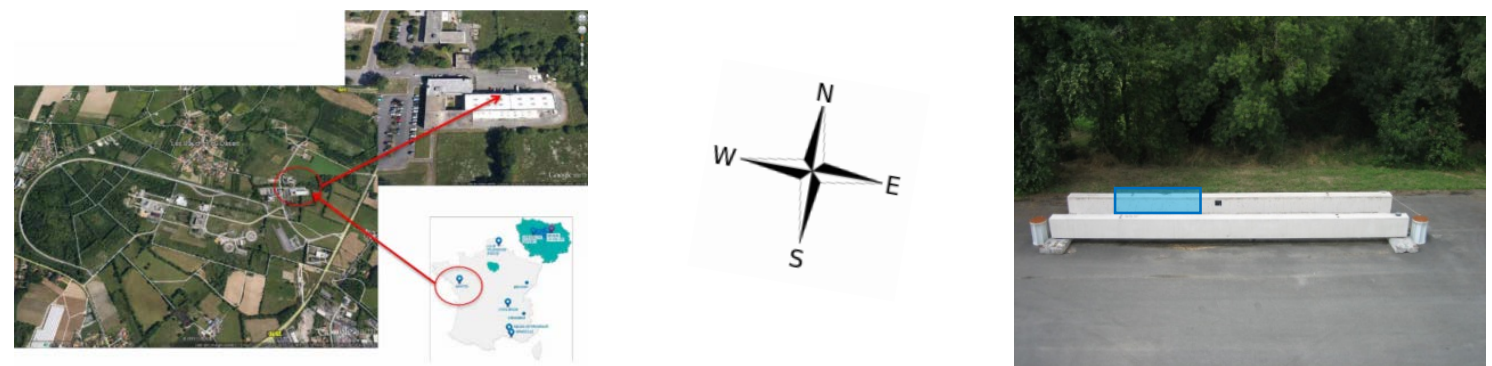

Fig. 5 Localisation of the outdoor test site (left) - view of the twin beams (right)

The beams are placed in front of a thermo-mechanical testing facilities building located at IFSTTAR center in Nantes. The monitoring system was implemented on the building roof facing the two elements. Moreover the two beams are placed right in front of the sun path. The healthy beam is placed on the foreground and the other one in the back ground, the blue area on the picture (see figure 5) point out the impacted zone.

The monitoring system is composed of the SensorBox, the weather station, a GPS unit, the infrared camera and a Wi-Fi access point.
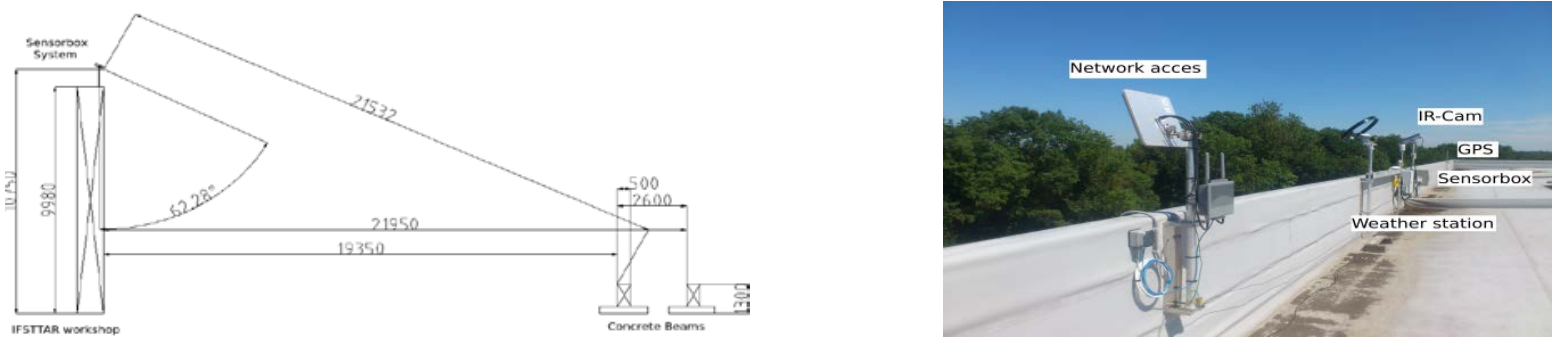

Fig. 6 Schematic view of the system and the roof layout

The infrared camera is placed in a thermally controlled caisson in order to protect it and insure a minimal temperature during the cold season. The front case is constituted of an optical adapted IR glass (with high transmission coefficient in the infrared wavelength used). The SensorBox is also implemented in a thermally controlled housing to prevent any overheating of the system during the warm season and freezing during cold season (see figure 6).

For the time being all measured data are backed up. Once they are on the storage server, post-processing at desired sub-sampling frequency can be made. The effect of the atmosphere is addressed through a transmission law that integrates environmental evolution with time. In the present paper, in a first approach, an average distance between the IR Detector and beams was taken to 20 meters (see figure 6) for the transmission evaluation with time.

\section{Experimental results}

Since august 2013 and until April 2014 nine months of data sampled at $0.1 \mathrm{~Hz}$ have been acquired with our designed system. One month break has been done in September for various maintenance operations and technical enhancement of the whole robustness of the system. 
During this interruption period, the optical lens of the infrared camera have been changed, from $10 \mathrm{~mm}$ focal length to $25 \mathrm{~mm}$ in order to increase the spatial resolution of the infrared image of the beams. Consequently, the monitoring of beam extremities in the longitudinal direction (parallel to beam) was no more possible.

\subsection{IR results}

Illustrations of thermal images acquired, with these two fields of view, at the beginning of the daily thermal relaxation phase and at its end, are shown in figure 7 . The infrared images also illustrate the gain in spatial resolution obtained by changing the camera optic.
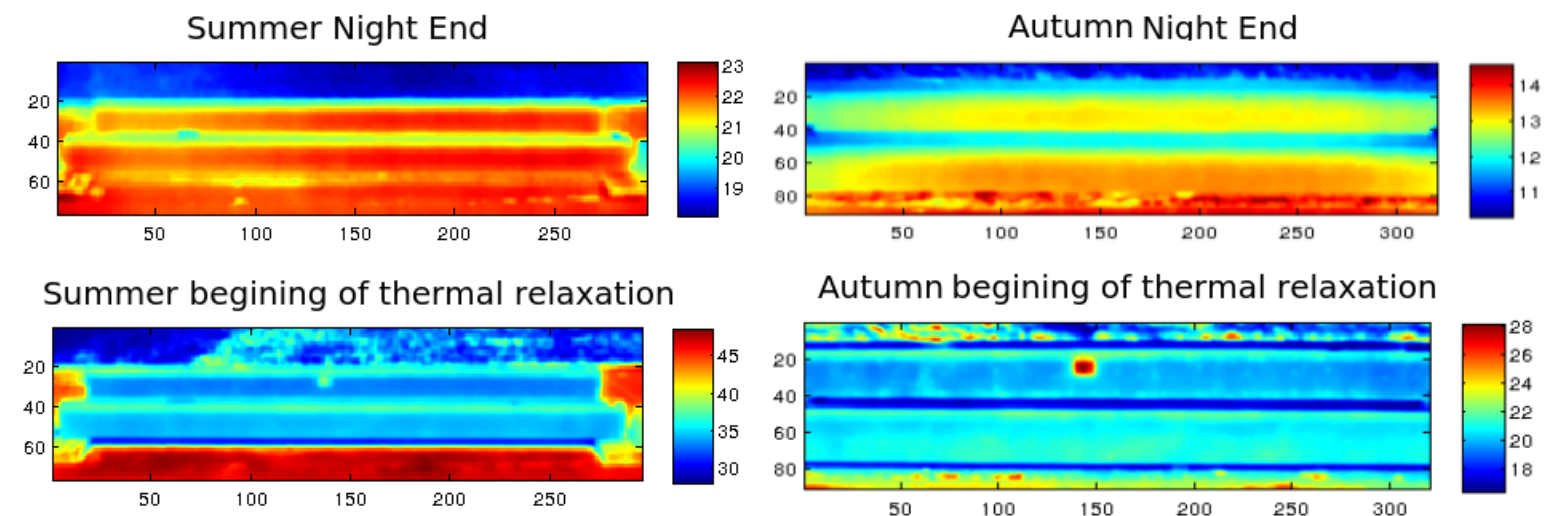

Fig. 7 Examples of infrared images at different moment of a day in two seasonal conditions

During those months, thermal images, air temperature, wind speed and direction, pressure, relative humidity, and rain variations with time have been measured. Figures 8 to 10 present the evolution during summer, autumn and the beginning of winter of these parameters.

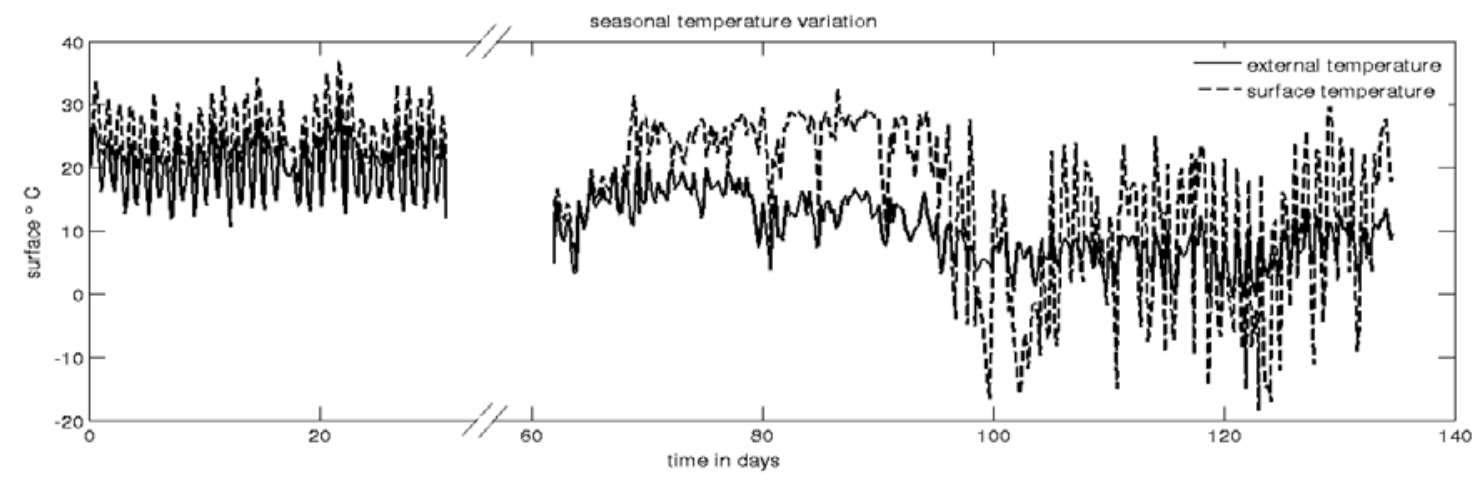

Fig. 8 Seasonal surface and external temperature variation

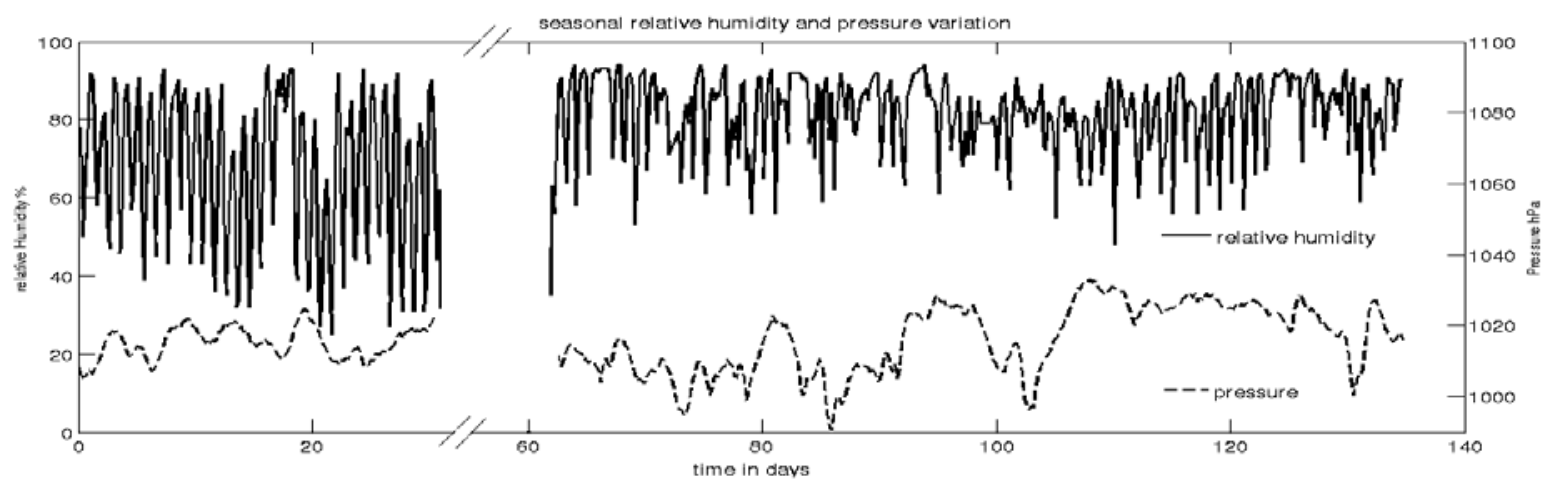

Fig. 9 Seasonal relative humidity and pressure variation 


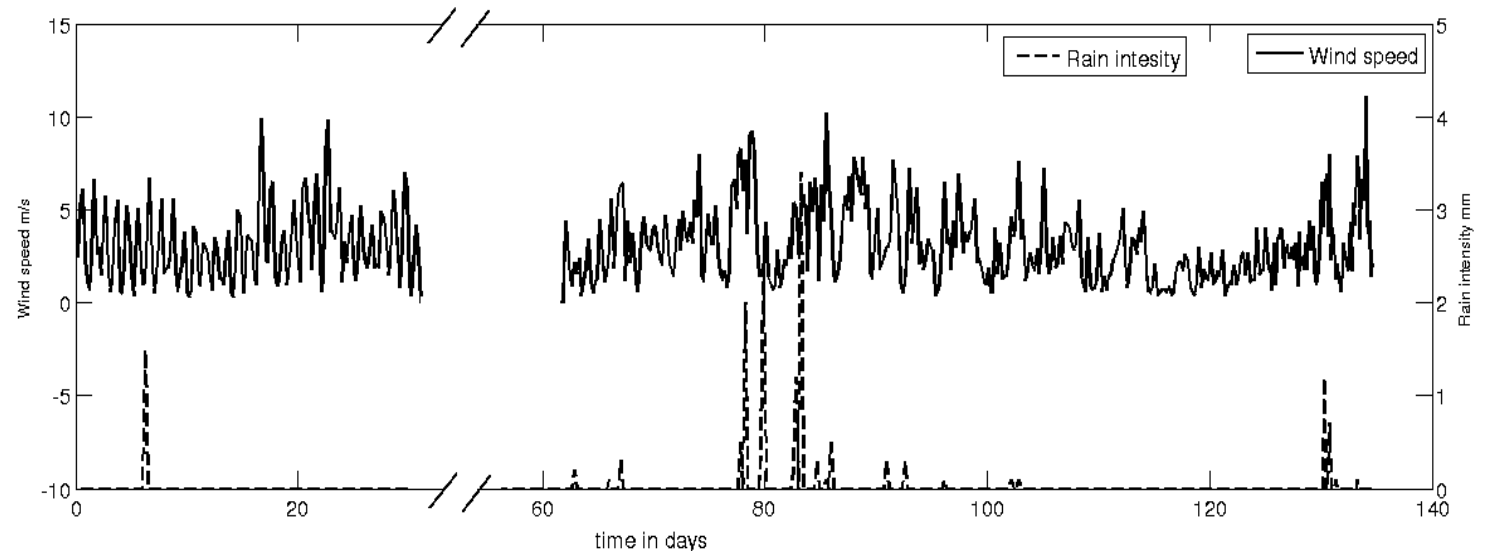

Fig. 10 Seasonal wind speed variation and rain intensity

These figures show the correlation that exists between meteorological parameters and the thermal value acquired by infrared thermography on the beam surface and also by the weather station used to monitor the atmosphere. For the thermal value the seasonal decay can also be observed. It has to be noticed that during the warm season all the parameters appears periodically, even for the wind where the daily periodic warming create a periodic air mass transfer. But, on the contrary during the cold season the effect of all the external perturbations, wind, nebulosity, building shadow (reducing direct solar illuminations up to no illumination during winter days), rain and heat flux seasonal decay combined make parameters evolution to be more randomly distributed.

From a physical point of view that means that we have to deal with two different systems. The first one is harmonically excited and the second appears quasi-randomly excited. This fact will have to be address when building physical models in particular when considering the boundary conditions but also when addressing possible signal processing approaches.

As this system is used to investigate the feasibility of a long term monitoring of structural elements and to cope with one of the objectives of this study, we reported in figure 11 the mean thermograms above a healthy and a defective area on the impacted beam, during one month period of the warm season. Unfortunately, due the high level of reinforcement of the studied beams effect of thermal diffusion tends to make uniform the thermal surface field, phenomenon that is also amplified by the reduced infrared thermography spatial resolution (see figure 11). Unlike the former study [8] where distinctive thermal diffusion behavior (inducing temperature field differences) were observed, here a standard heat transfer model used to minimize a cost function expressed in the meaning of the least square approach [11] might lead to inconsistent parameter estimation.

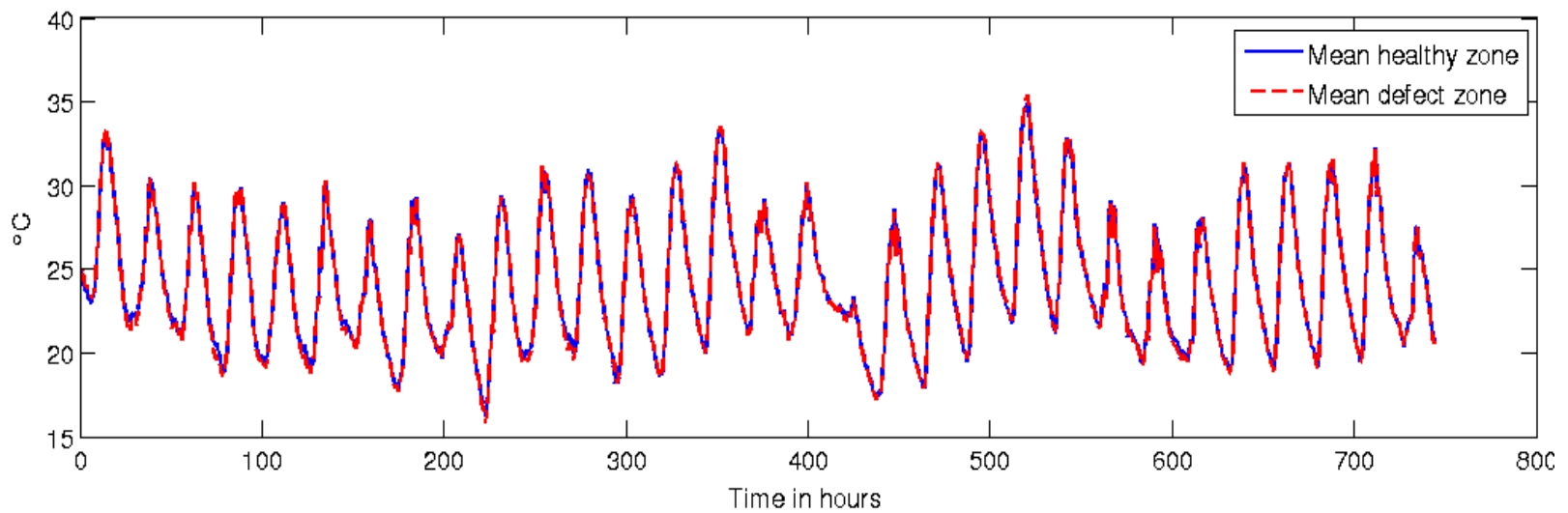

Fig. 11 Mean Thermograms on the impacted beam

In first months of 2014, some malfunctions at infrared camera level were observed, in particular bad thermal derivative compensation that finally drives us to stop the acquisition with the infrared camera for maintenance purpose. To pursue the measurements, a temporary solution has been found by replacing the defective FLIR SC 325 by a FLIR SC 655 with a $640 \times 480$ IRFPA with $17 \mu \mathrm{m}$ pixel pitch in the same spectral bandwidth and equipped with a $24 \mathrm{~mm}$ lens.

This unfortunate issue is a good example of the flexibility of the system indeed the operation takes place in few hours and didn't induce perturbation on the rest of the system.

Figure 12 presents an illustration of infrared image acquired with this new camera that has a better resolution and a better detector sensitivity. In figure 12 (right) a thermogramm over three days is also presented. 

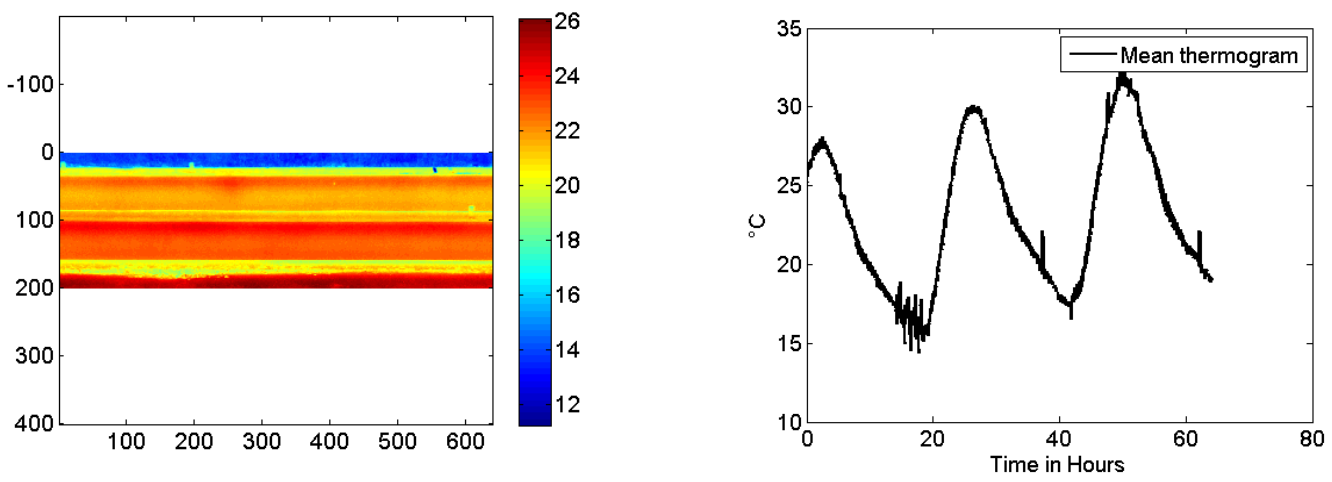

Fig. 12 Infrared image acquired at the sunset with the FLIR SC655 IR camera (left) - Thermogram issued from an area taken over the healthy beam viewed surface (right)

In the next paragraph data acquired through the instrumented beams are compared to those acquired with contactless sensing techniques previously presented for the 3 days period shown in figure 12 .

\subsection{Beam inner temperature analysis}

To complete the analysis presented in the previous paragraph using only external measurements, we compared them with data acquired with thermocouples located inside the healthy beam. We limited the analysis to the following of thermal field evolution in the beam during three days in May 2014, coupled with weather parameters and associated infrared measurements acquired in parallel. Figure 14 presents the weather evolution during those days. During that period atmospheric pressure decrease was observed.
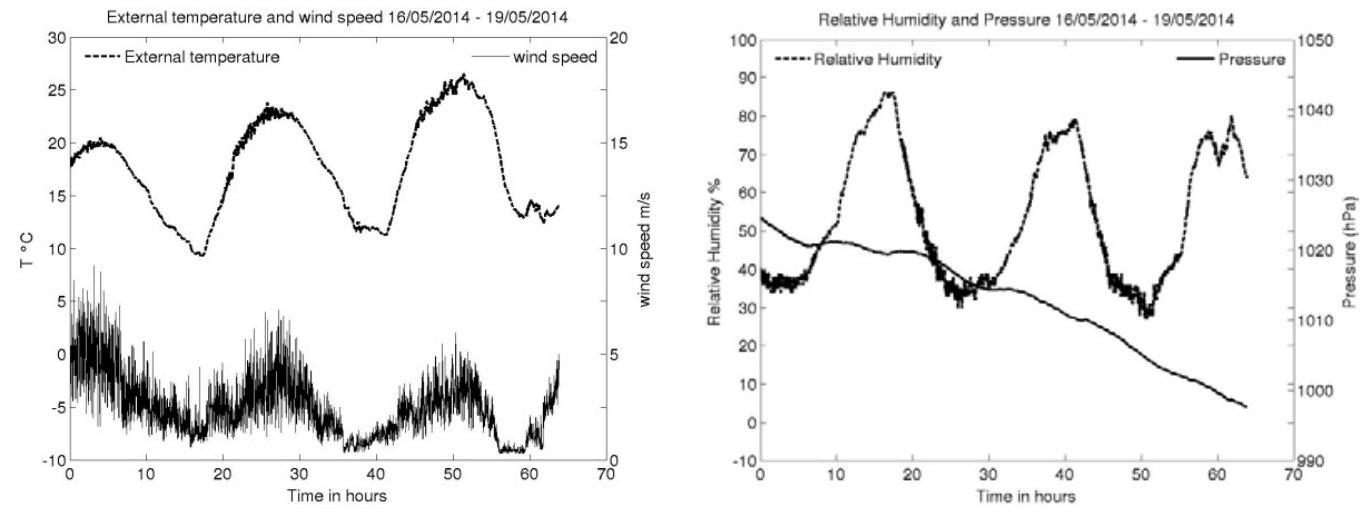

Fig. 13 Environmental parameters during three days

The thermocouples measurement protocol is divided into columns and lines (see figure 3). To compare data acquired by the Sensorbox with the thermocouple the study will be focus on section 9 and 7 of the beam that are located in the center of the field of view of our infrared camera. A first analysis shows that infrared measurements (see figure 12 right) are coherent with the thermocouples evolutions measured closer to the viewed surface (column $c^{3}$ row $r^{3}$ in figure 14).
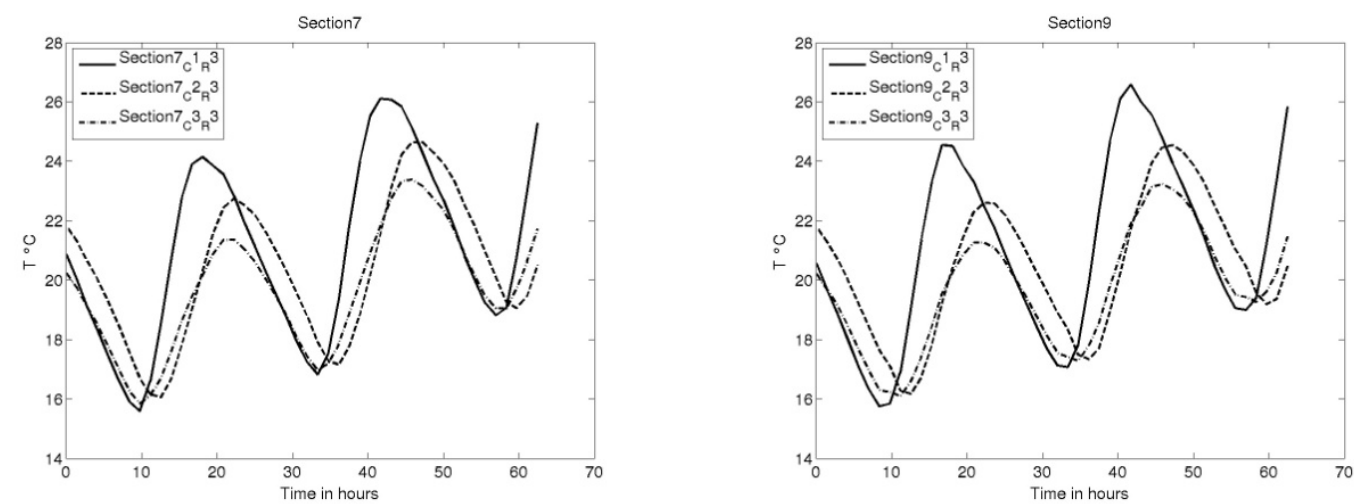

Fig. 14 Internal thermogram on the third row, section 7 (right) and section 9(left) 
The amplitude evolution along axes are in agreement with possible theoretical approaches [12]. The most interesting point is the emergence of a heat core $\left(\mathrm{C} 2 \_\mathrm{R} 3\right)$ in the beam in such environmental conditions. Its temperature signal is delayed versus the front face temperature but move from delayed to advance versus the rear face temperature. In fact according to the figure 14, even if only two faces (front and top) are directly submitted to the periodical solar heat flux, the both remaining faces are also submitted to periodical solicitations of smaller amplitude that also impact the global thermal behaviour of the structure.

The figure 15 show normalized heat flux computed with the Duffie and Beckman solar model [13] for the front face and the convective contribution calculated with the McAdams Correlation [14] for the rear face.

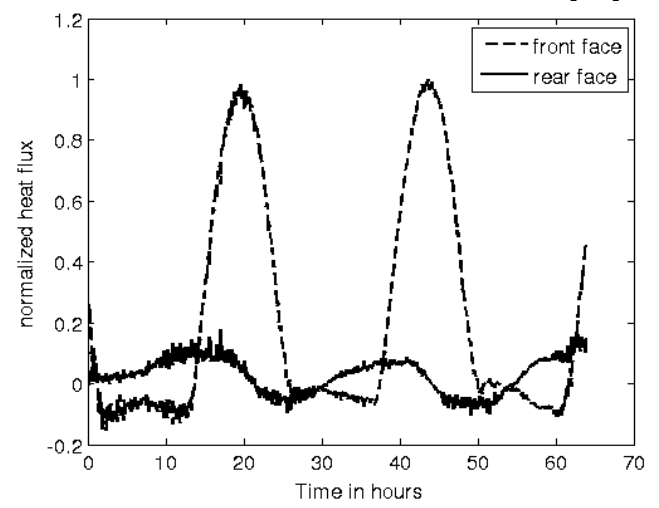

Fig. 15 Normalized heat flux

According to the figure 14 the rear face phase shift arise from the combination of the two boundary conditions (front and rear beam faces) thermal contribution and the conduction phase shift, the same goes for the phase shift between the upper face and the below one.

These observations points out the fact that the system appears to be always in transient regime when sun illumination affect the front face.

\subsection{FFT analysis}

In first approach FFT analysis was applied to thermal images sequence. Figure 16 shows results obtained on a thermal image sequence extracted over 10 days in the summer period matching the poorest spatial resolution for FLIR SC325 IR camera configuration.

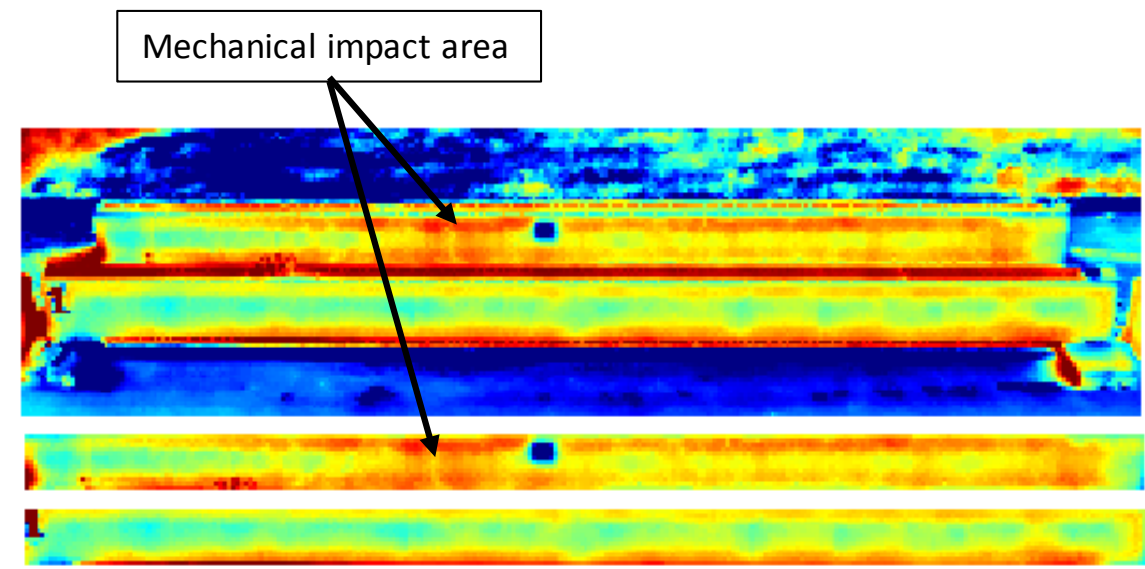

Fig. 16 Phase map of the Full IR scene focused on beam (top) - Phase map zoom on the damage beam (middle) Phase map zoom on the healthy beam (bottom)

A difference of phase values can be observed locally in the area where the mechanical impact affected the beam. The healthy beam does not present equivalent phase values at the same longitudinal position. The blue-dark square belongs to a reference area painted in black during falling block trials. 


\section{Conclusion}

We presented a thermal monitoring system based on recent advance in ICT technology to assess the very long term infrared monitoring of civil engineering structure.

The whole system is able to control an infrared camera as well as a weather station. Measurements are synchronized by a GPS clock. Developed in a first stage through the ISTIMES European project, the system has been enhanced in order to reach the full autonomy in term of power, data acquisition and backup. The system have been used to assess the long term monitoring of two large scale reinforced concrete beams. The first beam has been instrumented with 48 thermocouples and 10 strain gauges; the second one has been submitted to several high energetic mechanical impacts that have damage it. This twin beams are submitted to outdoor environmental condition since three years now. From the summer 2013 until now the infrared measurement system has acquired data. The system architecture itself has demonstrated its reliability.

Infrared and weather measurements led to show the correlation that exists between the temperature and the external conditions. Furthermore, two different thermal behaviour have been discriminated with results obtained. The first one corresponds to a system harmonically excited, the second one to a pseudo-random excitation.

A month of measurement has been especially studied where the system appears to be in established periodic regime. Due to the diffusion effect which tends to uniform the surface field, standard model used to minimize a cost function to estimate the beam parameters are here difficult to use.

The infrared measurement has been then compared to the inside beam thermocouples values. Those values have highlighted the presence of a heat core inside the beams. Furthermore, a classical FFT analysis allows us to put in evidence a difference of thermal answer in the impact area between the healthy and damage beam. Other location of damage are still open to processing method investigations.

One of the main perspectives of this study is to develop and embed into the Sensorbox a thermal model with an estimation procedure able to identify equivalent thermal properties and reconstruct the 3D internal temperature field by taking into account the environmental solicitations and their evolution with time.

\section{REFERENCES}

[1] Obran Z, Gutermann. Assessment of masonry arch railway bridges using non-destructive in-situ testing methods, Engineering Structures 31 pp 2287-2298, 2009. doi:10.1016/j.engstruct.2009.04.008.

[2] Rhazi N, Naar S. Aptitude de la thermographie infrarouge à détecter les fissures et nids d'abielle dans le béton 12e Colloque sur la progression de la recherche québécoise sur les ouvrages d'art 2005.

[3] Dumoulin J., Feuillet V., Marchetti M., Sauvagnat T., Ibos L. et Mazioud A. Evaluation de méthodes de détection de défauts, par thermographie infrarouge active, dans des échantillons d'enrobés bitumineux. SFT 2009 Vannes France.

[4] Clarck M R, et al. Application of infrared thermography to the non-destructive testing of concrete and masonry bridges. NDT\&E International Vol 36 pp 265-275, 2003. PII: S0963-8695(02)00060-9.

[5] Grinzato E et al. Quantitative infrared thermography in buildings. Energy and Buildings Vol 29 pp 1-9 1998.

[6] Proto M. et al. Transport infrastructure surveillance and monitoring by electromagnetic sensing: the ISTIMES project. Sensors, 10,10620-10639, 2010, doi: 10.3390/s101210620.

[7] Dumoulin J., Averty R. Development of an infrared system coupled with a weather station for real time atmospheric corrections using GPU computing: Application to bridge monitoring, in Proc of $11^{\text {th }}$ International Conference on Quantitative InfraRed Thermography, Naples Italy, 2012.

[8] Dumoulin J., Crinière A. and Averty R. Detection and thermal characterization of the inner structure of the "Musmeci" bridge deck by infrared thermography monitoring. Journal of Geophysics and Engineering, Volume 10, Number 2, November 2013, IOP Science, doi:10.1088/1742-2132/10/6/064003.

[9] Catapano I. et al Structural monitoring via microwave tomography-enhanced GPR: the Montagnole test site. Journal of Geophysics and Engineering, Volume 9, Number 4, August 2012, pp 100-107, IOP Science, doi:10.1088/1742-2132/9/4/S100.

[10] Nassiopoulos A. Identification rapide de la température dans les structures du genie civil. Thèse de doctorat Ecole nationale des ponts et chaussées 2008.

[11] Beck JV, Arnold KJ.1997. Parameter estimation in engineering and science. Wiley; New York (NY).

[12] Carslaw HS, Jaeger JC, Conduction of heat in solids. New York (NY): Oxford Science; 1959.

[13] Duffie, J. A., Beckman, W. A.. Solar Engineering of Thermal Processes, Wiley \& Sons, New York 1991.

[14] McAdams, W. H., Heat Transmission, McGraw-Hill, New York. 1954 